\section{Trastorno por déficit de atención e hiperactividad en niños aymara: primera aproximación clínica}

\author{
XIMENA CARRASCO ${ }^{1, \mathrm{a}}$, FRANCISCA DAIBER ${ }^{2, \mathrm{~b}}$, \\ PAULA ROTHHAMMER ${ }^{2, \mathrm{c}}$, DANIELA HUERTA ${ }^{2, \mathrm{~d}}$, \\ CLAUDIA ANDRADE ${ }^{2,5, \mathrm{e}}$, PATRICIA OPAZO ${ }^{2, \mathrm{f}}$, LIZA PAZ LAGOS ${ }^{3, \mathrm{~g}}$, \\ FRANCISCO ROTHHAMMER ${ }^{3,4, \mathrm{~h}}$, FRANCISCO ABOITIZ ${ }^{2,5, \mathrm{~h}}$
}

\section{Attention deficit hyperactivity disorder in Aymara children}

Background: The assessment of Attentional Deficit Hyperactivity Disorder (ADHD) among ethnic groups may reveal environmental or cultural variables that influence the appearance of this disorder. Aim: To assess the presence and characteristics of ADHD in two communities of the inland Arica valleys (Azapa and Lluta), where the Aymara population predominates. Material and Methods: Starting from a screening based on the Conner's test, we evaluated 79 children aged 8 to 13 years. Sixty children were of Aymara origin and 19 children were of non-Aymara origin. Twenty Aymara and 9 non-Aymara children had ADHD. They were compared with a group of patients from Santiago, Chile (110 children) that were previously assessed. Results: Patients from Azapa/Lluta displayed similar characteristics to those from Santiago. However the former had significantly less psychiatric comorbidities than the latter. On the other hand, the non-Aymara subgroup of Azapal Lluta displayed an increased rate of comorbidities and was exclusively of the combined subtype, although their sample size is too small to draw strong conclusions. Conclusions: Although we cannot dismiss biological variables, the importance of family values and the respect to authorities may be protective factors for ADHD, associated to Aymara culture. Our findings suggest that the clinical characteristics of ADHD are not uniform among ethnic groups and cultures. The relative contribution of environmental and genetic factors in this variability remain to be determined.

(Rev Med Chile 2012; 140: 1409-1416).

Key words: Attention deficit, disorder with hyperactivity; Child; Ethnic groups.

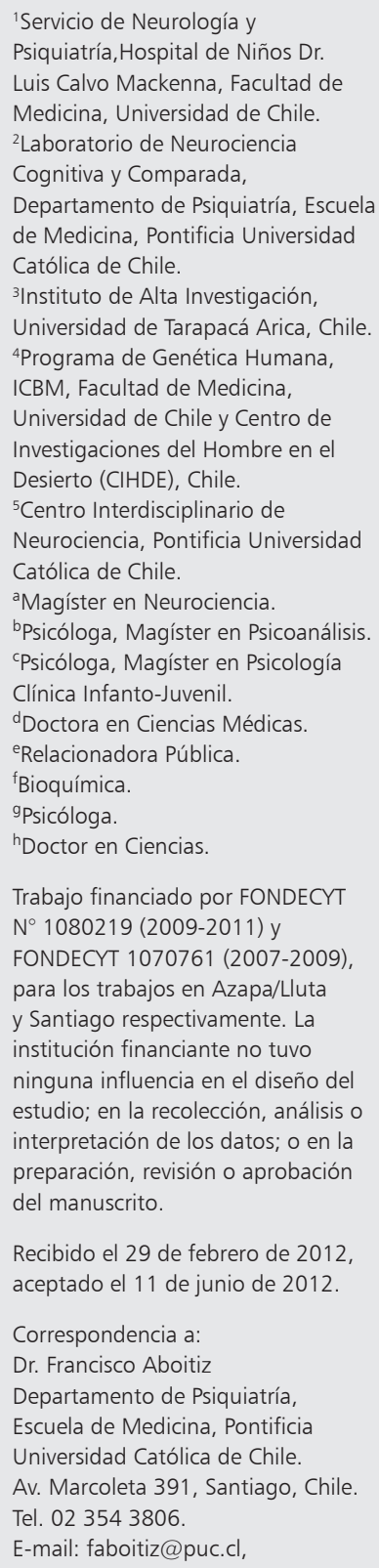

Recibido el 29 de febrero de 2012 , aceptado el 11 de junio de 2012.

Correspondencia a:

Dr. Francisco Aboitiz

Departamento de Psiquiatría, Escuela de Medicina, Pontificia Universidad Católica de Chile.

Av. Marcoleta 391, Santiago, Chile.

Tel. 023543806 .

E-mail: faboitiz@puc.cl

$\mathrm{E}$ 1 trastorno por déficit de atención e hiperactividad (TDAH) constituye una de las entidades neuropsiquiátricas más frecuentes en la edad escolar y ha sido diagnosticado en diversas culturas y etnias, estimándose su prevalencia entre 5 y $12 \%$ en países industrializados ${ }^{1,2}$. En Chile, se ha reportado que afecta a $5 \%$ en la población entre 4 y 18 años de edad ${ }^{3,4}$. El presente artículo muestra el análisis de las características clínicas de sujetos diagnosticados con TDAH en una población aymara en el extremo norte de Chile. Constituye la continuación de una serie de trabajos orientados a determinar rasgos endofenotípicos y marcadores genéticos en distintos grupos étnicos del país ${ }^{5,6}$.

La investigación del TDAH en grupos étnicos originarios tiene importancia por diversas razo- 
nes. En primer término, es necesario hacer un adecuado enfrentamiento clínico de este trastorno, porque dadas sus significativas repercusiones para el desarrollo personal y social, puede contribuir a perpetuar la situación de pobreza en que tales comunidades se encuentran ${ }^{7}$. Refleja su importancia el hecho que el TDAH es considerado, para el Ministerio de Educación de nuestro país, dentro de las condiciones con "Necesidades Educativas Especiales", lo que significa que, en caso de diagnosticarse, implica para el niño reforzamiento pedagógico, algunos ajustes curriculares y evaluativos y eventualmente la intervención de otros profesionales vinculados al neuro-desarrollo infantil ${ }^{8}$.

En segundo lugar, reviste interés por tratarse de niños y adolescentes en un ambiente sociocultural peculiar, lo que puede incidir en la interpretación que se hace de las manifestaciones propias del TDAH y sus comorbilidades. Además, la mayoría de los artículos que sugieren una prevalencia similar del TDAH entre distintas culturas o etnias, provienen de ambientes urbanizados ${ }^{2}$, con exigencias sociales muy diferentes de las rurales.

En tercer lugar, es interesante indagar si los mecanismos fisiopatológicos y los factores etiológicos del TDAH, mayoritariamente genéticos, corresponden en los aymara a los hasta ahora propuestos en otros grupos humanos ${ }^{9-11}$. Ello es propósito de aspectos de nuestra investigación no expuestos en este reporte.

Un estudio previo de nuestro grupo analizó el "riesgo de TDAH" en escolares aymara y rapa-nui, en base a un instrumento de tamizaje para detectar posibles pacientes afectados por TDAH, como es la escala de Conners abreviada ${ }^{12}$. En esta prueba los niños aymara tuvieron puntajes promedio menores que los niños de Santiago y éstos, a su vez, menores que los niños rapa-nui. También se encontró una distribución diferenciada de los alelos de riesgo de TDAH entre los tres grupos, destacando la mayor presencia del alelo DRD4-2R en los niños rapa-nuí.

Pese a ser una valiosa herramienta, la escala de Conners no permite hacer el diagnóstico de TDAH. La confirmación de este trastorno es clínica y personalizada y debe estar a cargo de uno o más profesionales con sólida formación en neuropsiquiatría infanto-juvenil, que puedan descartar correctamente diagnósticos diferenciales y determinar también comorbilidades ${ }^{2,13}$.
En el marco anterior, el objetivo del presente reporte es mostrar la estrategia y los resultados preliminares de la incursión a terreno de un equipo de profesionales en colegios con alta proporción de niños aymara en la Región de Arica y Parinacota, con el fin de diagnosticar TDAH, establecer sus principales características y determinar si existen diferencias significativas con respecto a niños chilenos no-aymara del mismo entorno y niños chilenos de la ciudad de Santiago.

La hipótesis subyacente es que existe TDAH en la población de escolares aymara, aunque exhibe diferencias significativas en su forma de presentación clínica, en comparación con el mismo trastorno en niños de otro origen étnico.

\section{Metodología}

\section{Sujetos}

Escolares de 8 a 13 años, de ambos sexos, de $3^{\circ}$ a $8^{\circ}$ básico, residentes en la Región de AricaParinacota, que asisten a dos establecimientos educacionales: Liceo Agrícola José Abelardo Núñez, de la localidad de San Miguel de Azapa, en el valle de Azapa, y Escuela G-8, Carlos Condell de la Haza, del valle de Lluta. En ambos colegios la población de niños de ascendencia aymara corresponde al $80 \%$. Todos los estudiantes son residentes de los respectivos valles y sus inmediaciones. Se incluyeron en el estudio tanto niños aymara como chilenos no-aymara. El nivel socioeconómico era mayoritariamente bajo y en algunos casos medio-bajo.

La ascendencia étnica de los niños se determinó en base a tres fuentes: apellidos, información aportada por los padres y datos de etnicidad consignados en los establecimientos educacionales respectivos. Se consideraron de etnia aymara aquellos niños en quienes se corroboraba al menos un antecesor aymara en línea directa.

Se contó además con una base de datos de 110 pacientes del policlínico de TDAH del Servicio de Neurología y Psiquiatría del Hospital Luis Calvo Mackenna, de igual rango etario y escolaridad, chilenos de etnia mixta, de ambiente urbano y nivel socioeconómico medio y medio-bajo, que está a cargo de una de las autoras (Carrasco X).

\section{Procedimiento diagnóstico:}

\subsection{Tamizaje mediante de escala de Conners abreviada}

La escala de Conners abreviada es el instru- 
mento más ampliamente difundido en nuestro país como método de tamizaje de TDAH y de seguimiento de los pacientes ${ }^{12}$. Consta de 10 ítems que indagan sobre síntomas cardinales de TDAH y que se califican entre 0 y 3 , en orden de severidad. Pese a ser una versión acortada, está escala muestra muy buen poder predictor de la presencia o ausencia del trastorno.

Los profesores jefes de $3^{\circ}$ a $8^{\circ}$ básico de ambos establecimientos, contestaron la escala de Conners abreviada para cada uno de sus alumnos. Las encuestas fueron recolectadas y enviadas al equipo de investigadores en Santiago.

Sólo dos de los 10 ítems de la escala abreviada de Conners apuntan a inatención; los restantes se refieren principalmente a indicadores de hiperactividad, impulsividad y desregulación emocional. De allí que el subtipo inatento de TDAH podría encontrarse subestimado si se analizan sólo puntajes totales en test de Conners; es necesario valorar cada ítem, individualmente. Por tal razón, se seleccionaron a quienes puntuaron más de 15 en todos los ítems del test y a quienes tuvieron puntajes " 3 " en al menos dos de los seis primeros ítems (entre los que se incluyen los dos ítems alusivos a inatención), como probables pacientes TDAH. Por otra parte, quienes puntuaron entre 0 y 10 , sin tener puntaje 2 ó 3 en ningún ítem, fueron preseleccionados como probables controles sin TDAH (para otros propósitos de comparación, no pertinentes a este artículo).

\subsection{Evaluación clínica en terreno}

El grupo clínico de investigadores realizó tres visitas a la Región de Arica-Parinacota: en junio de 2010 y marzo de 2011 para trabajar en el colegio del valle de Azapa, y en agosto de 2011 para trabajar en el colegio del valle de Lluta. Se utilizaron dependencias facilitadas por los equipos docentes de los propios establecimientos.

A partir de la preselección hecha de "probables TDAH" y "probables Controles", tanto aymaras como chilenos no-aymara, se procedió al algoritmo de recolección de sujetos que se ilustra en la Figura 1, donde se muestra además la firma de los consentimientos y asentimientos informados.

Debemos aclarar que el propósito del equipo era encontrar muestras pareadas para análisis electrofisiológicos y genéticos (no pertinentes a este artículo): 40 sujetos con TDAH (20 aymara y 20 chilenos no-aymara) y 40 sujetos de desarrollo normal (20 aymara y 20 chilenos no-aymara). En consecuencia, no es un diseño que sirva estrictamente para estimar prevalencia de TDAH.

El proceso de evaluación diagnóstica incluyó: - Entrevista y examen en privado a cada niño o niña con al menos uno de sus padres o apoderados, por médico neuropediatra especialista en trastornos del desarrollo y conducta.

- Entrevista semiestructurada por psicóloga clínica infanto-juvenil, utilizando la batería MINI-Kid para diagnóstico de morbilidades psiquiátricas ${ }^{14}$. - Aplicación de la escala de inteligencia de Wechsler para niños revisada WISC- $\mathrm{R}^{15}$ por una psicóloga clínica.

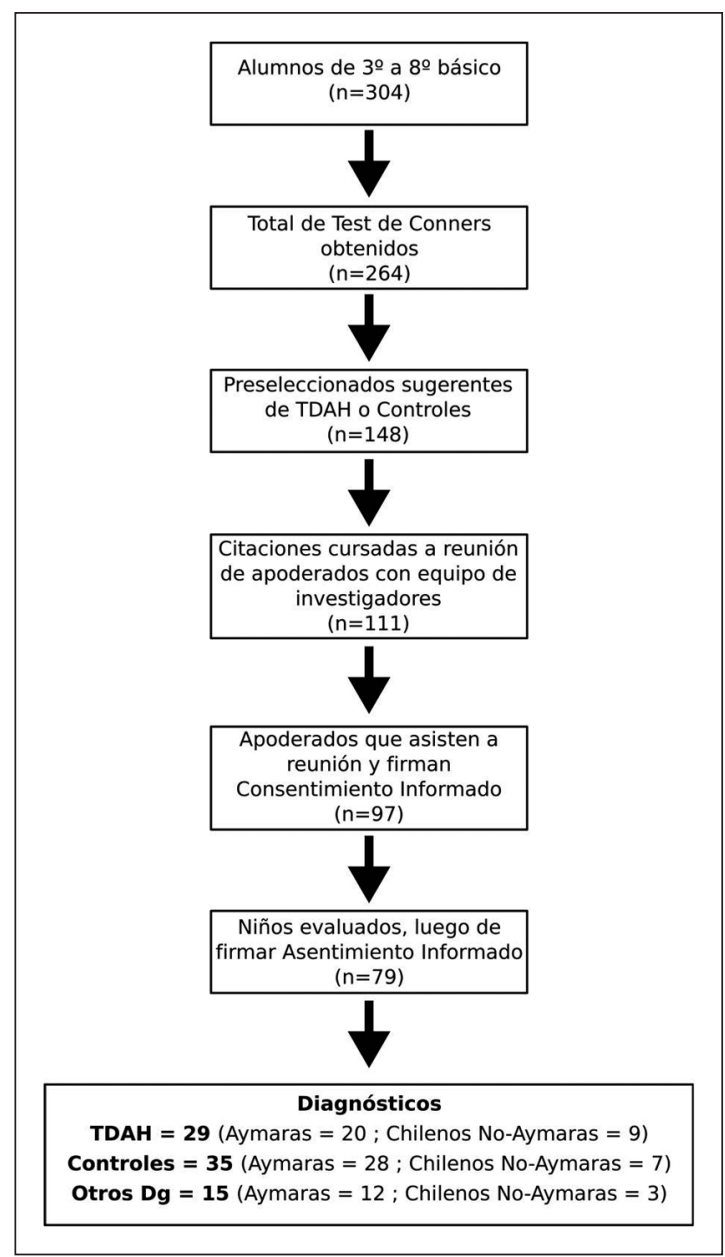

Figura 1. Metodología de enrolamiento de sujetos para el estudio en los valles del interior de Arica. Se presenta la suma de los sujetos de ambos colegios visitados: Liceo Agrícola José Abelardo Núñez, de San Miguel de Azapa y Escuela G-8, Carlos Condell de la Haza, de Valle de Lluta. 
Para el diagnóstico de TDAH se utilizaron los criterios del DSM-IV ${ }^{16}$, como asimismo para la distinción de subtipos (Combinado, Inatento e Hiperactivo/Impulsivo).

Este proyecto fue aprobado por los comités de ética de CONICYT, del Hospital Luis Calvo Mackenna y de la Escuela de Medicina de la Pontificia Universidad Católica de Chile.

\section{Resultados}

En base a las entrevistas en terreno en ambos establecimientos, se obtuvo el diagnóstico de TDAH en 29 niños: 20 de ascendencia aymara y 9 chilenos no-aymara. Por otra parte, se diagnosticaron 28 niños aymara y 7 niños chilenos no-aymara de desarrollo normal, sin TDAH. No se alcanzó la meta de 20 niños chilenos no-aymara por grupo; entre los 16 logrados, se detectaron más casos con TDAH y comorbilidades que controles ( 9 versus 7 sujetos). A todos ellos sumamos los datos de los niños chilenos del policlínico de Santiago.

La distribución por edad y sexo de los niños diagnosticados se presenta en la Tabla 1. No hay diferencias por género, predominando los hombres en los tres grupos $\left(\square^{2} 1,035 ; p=0,59\right)$. Sí hay diferencias en edad, por cuanto es significativamente mayor el grupo de chilenos no-aymara de Azapa/Lluta respecto a los otros dos grupos (Kruskal-Wallis, $\mathrm{p}=0,039$ ).

Los subtipos de TDAH diagnosticados se presentan en la Tabla 2. El subtipo combinado resulta mayoritario en los tres grupos, alcanzando al 100\% entre los chilenos no-aymara de Azapa/ Lluta, mientras que en los aymara, el subtipo inatento resulta discretamente más frecuente (40\%). Las diferencias no son estadísticamente

Tabla 1. Características demográficas de la muestra de niños evaluados en los valles de Azapa y Lluta y Santiago

\begin{tabular}{|lcccc|}
\hline & & $\begin{array}{c}\text { Aymara } \\
\text { Azapa/Lluta }\end{array}$ & $\begin{array}{c}\text { Chilenos } \\
\text { no-aymara } \\
\text { Azapa/Lluta }\end{array}$ & $\begin{array}{c}\text { Chilenos } \\
\text { Santiago }\end{array}$ \\
& $\begin{array}{l}\text { Sexo femenino } \\
\text { Edad promedio en años (DS) }\end{array}$ & $5(25 \%)$ & $3(33,3 \%)$ & $22(20 \%)$ \\
& Mediana de edad en años (IQR) & $9,55(1,82)$ & $11,33(1,12)$ & $9,98(1,91)$ \\
Total niños C/TDAH & $9(3)$ & $12(2)^{*}$ & $10(3)$ \\
Niños con otras morbilidades neuropsiquiátricas & 20 & 9 & 110 & NC \\
Niños control & 12 & 7 & NC \\
Total de niños evaluados & 28 & 19 & 110 \\
\hline
\end{tabular}

Los niños de Azapa/Lluta fueron diagnosticados en terreno, en sus respectivos colegios. Los niños de Santiago fueron pacientes atendidos en el Servicio de Neurología y Psiquiatría del Hospital de Niños Luis Calvo Mackenna en el período 2007-2009. No hay diferencias estadísticamente significativas en la proporción de géneros $\left(\square^{2} 1,035 ; p=0,59\right)$. La edad de la muestra de Chilenos No-Aymara de Azapa/Lluta (de pequeño tamaño y sin distribución normal), fue comparada mediante estadística no-paramétrica (Kruskal-Wallis); se observa que es significativamente de mayor edad respecto a los otros dos grupos $(p=0,039)\left({ }^{*}\right)$. No hay diferencias de edad entre los Aymara y los Chilenos de Santiago ( $T$ de Student -0,97; $p=0,341$ ). DS = Desviación estándar; $\mathrm{IQR}=$ Intervalo intercuartílico. NC = No corresponde.

Tabla 2. Subtipos de TDAH según grupo

\begin{tabular}{|lccc|}
\hline & $\begin{array}{c}\text { Aymara } \\
\text { Azapa/Lluta }\end{array}$ & $\begin{array}{c}\text { Chilenos } \\
\text { No-aymara Azapa/Lluta }\end{array}$ & $\begin{array}{c}\text { Chilenos } \\
\text { Santiago }\end{array}$ \\
\hline TDA combinado & $12(60 \%)$ & $9(100 \%)$ & $83(75,45 \%)$ \\
TDA predominio Inatento & $8(40 \%)$ & $0 \quad(0 \%)$ & $27(24,55 \%)$ \\
Total & 20 & 9 & 110 \\
\hline
\end{tabular}

No se observan diferencias significativas en la proporción de subtipos $\left(\square^{2} 5,38 ; p=0,06\right)$. 


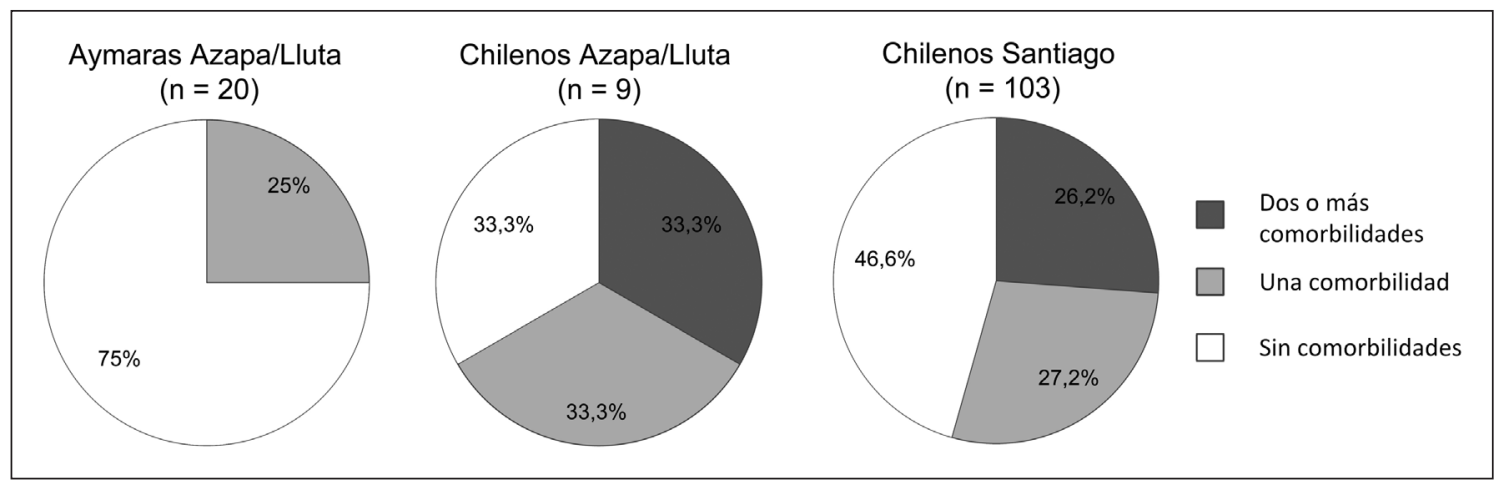

Figura 2. Presencia de comorbilidades psiquiátricas en niños con diagnóstico de TDAH, según grupos. La comparación de proporciones [Comorbilidades (+)/Comorbilidades (-)] da una diferencia estadísticamente significativa en cuanto el grupo Aymara presenta menos comorbilidades $\left(\square^{2} 6,47 ; p=0,039\right)$.

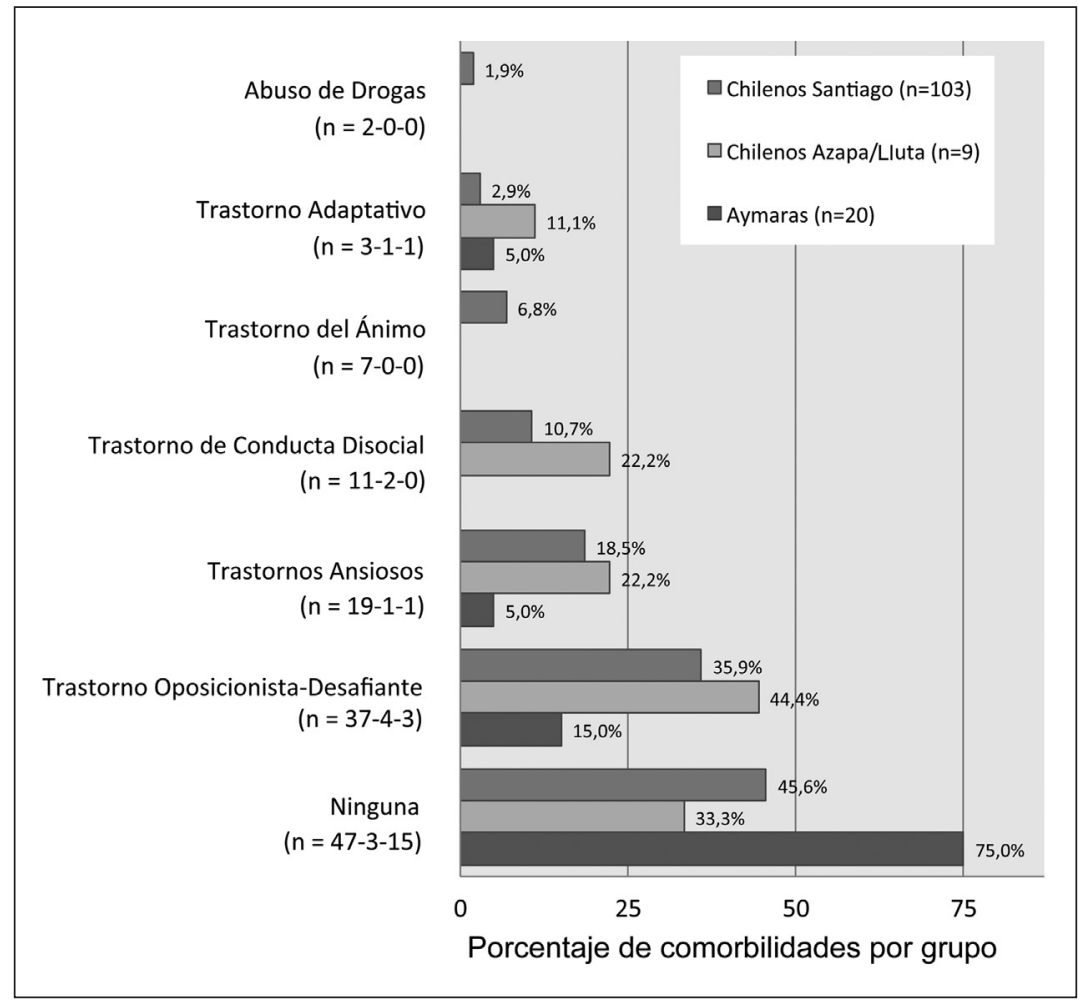

Figura 3. Tipos de comorbilidades psiquiátricas detectadas en niños con diagnóstico de TDAH, según grupo. Porcentajes calculados sobre el total de niños de cada grupo. Existen sujetos con 2 o más comorbilidades, por lo que puede excederse el $100 \%$. Entre paréntesis en cada diagnóstico de comorbilidad, el número de pacientes de los tres respectivos grupos.

significativas $\left(\square^{2} 5,38\right.$; $\left.p=0,06\right)$. El subtipo hiperactivo/impulsivo no ha sido encontrado en ningún grupo.

La presencia de comorbilidades por individuo es significativamente más baja en el grupo de niños con TDAH aymaras (25\%), en relación con los niños con TDAH chilenos no-aymaras de Azapa/ Lluta $(66,7 \%)$ y chilenos de Santiago $(53,4 \%)$, lo que se ilustra en la Figura $2\left(\square^{2} 6,47 ; p=0,039\right)$. Se aprecia además que en el grupo aymara sólo encontramos comorbilidades aisladas, mientras que en los otros dos grupos se encuentran casos con más de una comorbilidad.

Una comparación de los tipos específicos de comorbilidad y su magnitud relativa en los tres grupos estudiados se ilustra en la Figura 3. 


\section{Discusión}

Este estudio establece, hasta donde sabemos, la primera descripción clínica de la presentación de TDAH en una muestra de niños pertenecientes a la etnia aymara residente en nuestro país. El reporte muestra un avance de un estudio a escala mayor, donde la recolección de la información de los pacientes se basa en un exhaustivo trabajo clínico llevado a cabo por especialistas. Una importante dificultad ha sido lograr un grupo control de chilenos no-aymara, residentes en las mismas localidades, que contribuya a discriminar mejor el rol de variables ambientales (como la condición rural y regional), de variables étnico/culturales y biológicas ${ }^{17}$. Hasta el momento hemos reclutado sólo a 16 sujetos de este grupo, 9 con TDAH. Por tal razón, las comparaciones más confiables surgen del grupo aymara y el grupo de pacientes de Santiago, aunque igualmente consideramos los hallazgos en el reducido grupo de chilenos no-aymara de Azapa/Lluta.

Empleando la metodología estándar de entrevista clínica en profundidad y reportes de diversas fuentes, se evidencian casos con TDAH combinado e inatento entre los niños aymara de los valles interiores de Arica, lo que nos parece uno de los aspectos más valiosos de este trabajo. Ninguno de esos niños había sido diagnosticado antes, si bien los padres y profesores notaban importantes dificultades en su desarrollo escolar y psico-social en general. Las características clínicas y el relato de las madres respecto a las dificultades presentadas por sus hijos fueron similares a las que se tienen en pacientes de Santiago. Debemos destacar que la falta de diagnóstico significa que las políticas educacionales que deberían beneficiar a los escolares con $\mathrm{TDAH}^{8}$, no están siendo aplicadas. Lejos de existir un sobrediagnóstico de TDAH, en estas comunidades el subdiagnóstico (de éste y otros trastornos), parece ser el problema principal, lo que a su vez conlleva una subestimación de recursos materiales y humanos al momento de planificar intervenciones en salud y educación.

No se observaron diferencias significativas en cuanto a la distribución por subtipos de TDAH, aunque la proporción del subtipo inatento resultó algo mayor entre los niños aymara. A este respecto, se ha observado que éste puede ser el tipo predominante en estudios hechos en terreno, sobre población general, como es nuestra mues- tra de Azapa/Lluta. Las muestras de poblaciones clínicas, como la de Santiago, tienen un sesgo de sobre-representación de formas clínicas más disruptivas, como el TDAH combinado, tema que ha sido discutido por diversos autores ${ }^{18}$. Por lo mismo destaca, aunque son sólo 9 casos, que todos los chilenos no-aymara de Azapa/Lluta sean de tipo combinado. De acuerdo a la literatura, no extraña la ausencia del subtipo sólo hiperactivo 9 .

La alta tasa de comorbilidades en el pequeño grupo de niños chilenos no-aymara de Azapa/Lluta, incluso múltiples en un mismo sujeto, excede también lo observado en el grupo de Santiago. Las razones son imposibles de dilucidar con el presente estudio, sin embargo, nos permitimos señalar que se trata de niños que viven en condiciones de pobreza, miembros de familias que optan por residir en un ambiente donde los individuos de etnia aymara son mayoritarios, tras lo cual se esbozan complejos factores bio-psico-sociales ${ }^{19}$. Es además un grupo significativamente de mayor edad respecto a los aymara y a los chilenos de Santiago, lo cual también puede contribuir a una más alta tasa de comorbilidades ${ }^{2}$.

A la inversa, nuestros datos muestran que los niños aymara con TDAH tienen significativamente menor número de comorbilidades psiquiátricas que los otros grupos, siendo éstas las habituales: Trastorno Oposicionista-Desafiante, Trastorno Ansioso y Trastorno Adaptativo (un caso de cada uno) $)^{2}$. Ya que ésta es una muestra no-sesgada, lo anterior podría deberse a que las características étnico-culturales aymara sirven de alguna manera como factores protectores. La ruralidad no parecería ser el único factor protector, si consideramos que el reducido grupo de niños con TDAH chilenos no-aymara, también rural, tiene muy alta tasa de comorbilidades. En línea con esta interpretación creemos necesario reportar las observaciones cualitativas sobre los aymaras que nos resultaron más categóricas:

1. Alta valoración de la familia.

2. Padres con gran dedicación a sus hijos, que muestran preocupación por su desarrollo psicológico y capaces de entregar información precisa sobre las variables de salud interrogadas.

3. Relación explícitamente afectuosa de madres y padres hacia sus hijos y preocupación recípro$\mathrm{ca}$, de los hijos hacia sus progenitores, que en ocasiones planteó el diagnóstico diferencial de "ansiedad de separación", pero nunca alcanzó 
la categoría de trastorno.

4. Individuos con temperamento mayoritariamente contemplativo y sereno. Adultos con un estilo de vida austero, dedicados casi exclusivamente al trabajo agrícola y pastoril.

5. Gran orgullo de su etnia y tradiciones.

Al margen de lo anterior, lamentablemente también observamos muchas familias en condición de pobreza extrema, con alcoholismo entre los padres y/o con una relación autoritaria de los padres, especialmente hacia sus esposas.

Las observaciones antes enumeradas concuerdan con la literatura sociológica respecto al pueblo aymara: Aunque hay una adaptación a una forma de vida occidental, la cultura aymara se caracterizaría por un tipo de pensamiento pre-colonial, donde prevalece un sentido comunitario y de reciprocidad intrafamiliar. Destacarían la falta de individualismo, el respeto a la autoridad, la valoración de la honestidad, un profundo sentimiento mágico-religioso y la conexión con la tierra ${ }^{20,21}$. En este marco, los niños diagnosticados por nosotros podrían manifestar menos rasgos oposicionistas y sentir mayor necesidad de contención afectiva, lo que incidiría en la ocurrencia de elementos de ansiedad de separación y en la inhibición de conductas disruptivas.

Como conclusión, hemos detectado casos de TDAH equivalentes a los de Santiago entre escolares aymara de los valles interiores de Arica. Nuestros hallazgos apuntan a que los componentes étnicos o sociales, o ambos, tienen un impacto sobre su manifestación clínica, específicamente respecto a una menor tasa de morbilidades psiquiátricas asociadas. Contribuimos así a la idea que el TDAH no tendría una presentación uniforme a través de las etnias, existiendo dimensiones genéticas y/o culturales con incidencia sobre la expresión de sus síntomas. Un estudio previo de nuestro grupo, mostró una diferencia importante en los indicadores de TDAH entre las poblaciones rapa-nui y aymara, sugiriendo un rol de factores genéticos ${ }^{6}$. Esperamos poder sacar conclusiones más definitivas en la medida que se obtenga una muestra mayor. Finalmente, debemos señalar que el acceso de las comunidades aymara a profesionales de la salud es una necesidad pendiente, que las familias y los profesores con quienes trabajamos en este estudio nos manifestaron explícitamente.

Agradecimientos: Agradecemos la colabora- ción prestada por los directores de los colegios visitados, don Guillermo Fernández Canque, de ascendencia aymara, $\mathrm{PhD}$ en Educación, del Liceo Agrícola José Abelardo Núñez de San Miguel de Azapa, valle de Azapa y don Nicolás Montecinos, $\mathrm{PhD}$ en Educación, de la Escuela Básica G-8, Carlos Condell de la Haza, del valle de Lluta. Agradecemos además a sus equipos docentes y paradocentes. Fuimos testigos del cercano y positivo vínculo existente entre los niños y sus profesores. Por último, queremos agradecer particularmente a los estudiantes de los establecimientos visitados y sus familias, quienes participaron de manera entusiasta y desinteresada en todas las instancias del estudio.

\section{Referencias}

1. Faraone SV, Sergeant J, Gillberg C, Biederman J. The worldwide prevalence of ADHD: Is it an American condition? World Psychiatr 2003; 2: 104-13.

2. Barkley RA. Attention-Deficit Hyperactivity Disorder: A handbook for diagnosis and treatment. Third. Edition. The Guilford Press, New York. 2006.

3. Vicente B, Navas L, Belmar M, Holgado FP. [Analysis of a scale for the assessment of attention deficit disorder with hyperactivity in Chilean children]. Rev Med Chile 2010; 138 (12): 1502-9.

4. Vicente B, De La Barra F, Saldivia S, Kohn R, Rioseco P, Melipillán R. Prevalence of child and adolescent psychiatric disorders in Santiago, Chile: A community epidemiological study. Soc Psychiatry Psychiatr Epidemiol. 2011, [Epub ahead of print].

5. Rothhammer F. [Genomic Medicine: A privilege of developed countries?]. Rev Med Chile 2005; 133 (9): 1108-10.

6. Lagos LP, Silva C, Rothhammer P, Carrasco X, Llop E, Aboitiz F, et al. Riesgo de déficit atencional/hiperactividad en escolares Aymará, Rapa-Nui y de Santiago de Chile. Posible contribución de polimorfismos genéticos del sistema dopaminérgico. Rev Med Chile 2011; 139: 600-5.

7. De la Barra F, Toledo V, Rodríguez J. Prediction of behavioral problems in Chilean schoolchildren. Child Psychiatr Hum Dev 2005; 35 (3): 227-43.

8. hltp://www.mineduc.cl/index2.php?id_portal=20\&id seccion=3084\&id_contenido=12269. Ley 20.201/2007. Decreto Supremo No 170/2009

9. Biederman J, Faraone SV. Attention-deficit hyperactivity disorder. Lancet 2005; 366: 237-48.

10. Faraone SV, Perlis RH, Doyle AE, Smoller JW, Goralnick 
JJ, Holmgren MA, et al. Molecular genetics of AttentionDeficit/Hyperactivity Disorder. Biol Psychiatr 2005; 57: 1313-23.

11. Durston S, de Zeeuw P, Staal WG. Imaging genetics in ADHD: A focus on cognitive control. Neurosci Biobehav Rev 2009; 33: 674-89.

12. MINSAL Chile. Guía Clínica sobre "Atención Integral de Niñas/Niños y Adolescentes con TDAH" (Anexo 1), 2008.

13. NIMH (National Institute of Mental Health). Attention Deficit Hyperactivity Disorder. NIMH Publication, 2004. Electronic version. http://www.nimh.nih.gov/ publicat/index.cfm.

14. Sheehan DV. M.I.N.I. KID 4.0, 2004 (Copyright 19982004, USA). Versión en Español (Colón-Soto M, Díaz $\mathrm{V}$, Soto O).

15. Adriazola A, Ascencio S, Bernales M, Carrasco C, Keller A, Polanco F, et al. Estandarización de la Escala Revisada de Wechsler para la medición de la inteligencia en los niños (WISC-R). Memoria para optar al título de Psicólogo. Pontificia Universidad Católica de Chile, Santiago de Chile, 1976.
16. American Psychiatric Association: Diagnostic and Statistical Manual of Mental Disease. Washington DC: American Psychiatric Association, 1994.

17. Rutter M. Psychosocial influences: Critiques, findings and research needs. Dev Psychopathol 2000; 12: 375-405.

18. Baeyens D, Roeyers H, Vande Walle J. Subtypes of Attention-Deficit/Hyperactivity Disorder (ADHD): Distinct or related disorders across measurement levels? Child Psychiatr Hum Dev 2006; 36: 403-17.

19. Carter AS, Wagmiller RJ, Gray SAO, McCarthy KJ, Horwitz SM, Briggs-Gowan MJ. Prevalence of DSMIV Disorder in a representative healthy birth cohort at school entry: Sociodemographic risks and social adaptation. J Am Acad Child Adolesc Psychiatr 2010; 49 (7): 686-98.

20. Alberty G, Mayer E. Reciprocidad e intercambio en Los Andes peruanos. Perú Problema Instituto de Estudios Peruanos) 1974; 12.

21. Llanque D. La cultura Aymara: Desestructuración o afirmación de identidad. Editorial Tarea. Asociación de publicaciones educativas, 1990. 